\title{
Probing local electronic property of two-dimensional materials by atomic force microscopy
}

\author{
Zegao Wang ${ }^{1,2}$, Mingdong Dong ${ }^{1 *}$ \\ 1) Interdisciplinary Nanoscience Center (iNANO), Aarhus University, DK-8000 Aarhus C, Denmark \\ 2) Department of Materials Science, Sichuan University, 601165 Chengdu, China \\ ${ }^{*}$ E-mail dong@inano.au.dk
}

Compared to zero-bandgap graphene, 2D layered transition metal dichalcogenides (TMDs) with the chemical formula $\mathrm{MX}_{2}$ (where $\mathrm{M}=$ group IVB-VIIB metal and $\mathrm{X}=$ chalcogen) have brought new possibility for the applications in ultralow-power electronics due to the reasonable bandgap. Currently, the carrier mobilities of TMDs at ambient conditions are a little bit low which limit their practical applications. Many strategies, for example synthesizing new member of 2D TMDs, revealing the transport mechanism and optimizing the device configuration are acknowledged as the promising methods. Except metallic and insulate property, many $2 \mathrm{D}$ layered materials exhibit semiconducting behavior including $\mathrm{n}$ type, p-type or ambipolar, which could be used to fabricate various electronics. Compared to unipolar (n-type or p-type) transistors, ambipolar transistors, which can easily switch between n-type and p-type behavior by applying an electric field, are most promising candidates since they can effectively simplify circuit design and save the layout area in CMOS.

In this presentation, scanning probe technique including electric field microscopy, kelvin probe force microscopy and current atomic force microscopy, will be introduced to study the local electronic property of 2D layered semiconductor. The surface potential, aligned energy band in p-n junctions and local carrier transport property of CVD-grown $\mathrm{PtSe}_{2}$ will be talked.[3-5] Furthermore, the dimensionality dependent ambipolar $\mathrm{WSe}_{2}$ will be introduced from locally field screening and tuned work function under external electric field.

References:

[1] Z. Wang, Q. Li, F. Besenbacher, M. Dong, Adv. Mater. 28, 10224 (2016).

[2] Z. Wang, Q. Li, Y. Chen., et al. NPG Asia Mater. 2018, Doi.org/10.1038/s41427-0180062-1

[3] Z. Wang, Q. Li, H. Xu, et al. Nano Energy 49, 634 (2018) 